tenue sera répartie par moitié entre la Suisse et le Grand Duché de Bade.

Le devis de ces travaux est évalué à 25 millions de francs. La Société de construction et d'exploitation qui s'est constituée en lévrier 1908 pour les faire exécuter (Kraftwerke Laufenburg) est au capital actions de 18 millions de francs, elle a son siège en Suisse, toutes les actions ont été prises par un syndicat germano-suisse où figurent notamment la Banque pour entreprises électriques et la Société de crédit suisse, toutes deux à Zurich.

Outre ces deux usines de force électrique d'Angst-Wylen ct Laufenbourg en construction, il faut signaler deux projets qui sont à l'étude depuis quelques années.

Le premier consiste à aménager la chute de Rheinan, à quelques kilomètres en aval de Schaffhouse dans une boucle formée par le Rhin. Il a fait en 1902 l'objet d'une demande de concession de la part d'un consortium composé de la municipalité de Winterthur, de la Société pour l'industrie de l'aluminium à Neuhausen et de la Société d'électricité, précédemment Schuckert et C1e, à Nuremberg. On utiliserait les eaux du Rhin sur un parcours de I I kilomètres qui donnerait une chute de II mètres; la force escomptée serait de 43000 chevaux. Les difficultés entre les cantons de Zurich et de Schalfhouse au sujet des droits de souveraineté sur la portion du fleuve envisagée dans le projet de concession ont retardé jusqu'à présent l'octroi de celle-ci par le gouvernement badois et les deux cantons intéressés.

A 20 kilomètres en aval de Rheinan se trouve un autre point, Eglisau, que la municipalité de Zurich a étudié dès I900 pour $y$ installer une usine hydro-électrique pouvant fournir une force moyenne de 32000 chevaux. A cet endroit, les deux rives du Rhin appartiennent au canton de Zurich, de sorte que la concession dépend de ce seul canton. Mais le projet définitif n'a pas encore été arrêté.

Plusieurs autres entreprises du même genre ont été envisagées entre Schaffhouse et Bâle, notamment à Schwœnstadt où la force recueillie (30000 chevaux) serait réservée au Grand Duché de Bade, à Birofelden un peu en amont de Bâle où elle serait destinée au canton de Bâle-Campagne, et à Petit-Huningue, quartier industriel de Bâle qui remplacerait volontiers ses machines à vapeur par des dynamos. Cependant, aucune d'elles ne paraît devoir être réalisée avant plusieurs années.

Il n'en est pas de même d'un projet qui cette fois intéresse non plus la Suisse et le Grand-Duché de Bade, mais l'Alsace, et en particulier la ville de Mulhouse. Ce projet, conçu par un ingénieur de Paris, M. René Kœchlin, en collaboration avec M. Potterat, de Berne, et la maison de construction Havestadt et Contag, de Berlin, a été soumis en I9O2 aux gouvernements d'Alsace-Lorraine et du Grand-Duché de Bade. Depuis lors, il a été modifié, puis, en Igo8, communiqué par un comité d'initiative formé à Mulhouse à deux experts, MM. Theodor Kuhn, de Berlin, et Ed. Locher-Freuler de Zurich, dont le rapport a été publié tout récemment.

Le projet, amendé par ces experts, comporte l'utilisation de la force motrice du Rhin en deux installations successives, la première comprenant la chute du fleuve depuis la frontière suisse jusqu'à Kembs sur la rive gauche, la seconde depuis Kembs jusqu'à Petit-Landau à Io kilomètres environ en aval. Cette dernière installation, qui ne doit être construite que lorsque la force de la première usine aura été placée, sera réalisée par le prolongement du canal de fuite de la première usine formant ainsi le canal d'amenée d'eau au bâtiment des turbines de la seconde installation.

Le système adopté est le même qu'à Rheinfeliden : un barrage mobile et un canal latéral. Le barrage se composerait de 6 travées de $30 \mathrm{~m}$. de portée, de manière à relever de $2 \mathrm{~m}$. 50 au-dessus du niveau actuel le niveau d'eau du Rhin, il disparaitrait entièrement en temps de crue. Le canal d'amenée aurait son entrée à $300 \mathrm{~m}$. en amont du barrage du côté alsacien, sa longueur serait de $6.460 \mathrm{~m}$., sa largeur et sa pro. fondeur normales de 86 et 5 mètres respectivement Le bâti. ment des turbines contiendrait 12 turbines à axe horizontal fournissant chacune 5000 chevaux aux basses eaux sous Io m. 85 de chute. Enfin, pour sauvegarder les droits de h navigation et sur la demande expresse de la "Commission centrale pour la navigation du Rhin ", on a prévu une grande écluse de $25 \mathrm{~m}$. de largeur sur $90 \mathrm{~m}$. de longueur (I).

Les experts estiment que lon pourrait ainsı réaliser de façon permanente une force de 45600 chevaux d'où 11 résulte qu'en admettant un rendement moyen de $9 \mathrm{I} \%$ seulement l'usine serait en mesure de livrer en nombre rond 30300 lifio watts. Avec l'appoint de la Centrale à vapeur de Mulhouse qui fournira I I ooo kilowatts, l'usine hydro-électrique do Kembs pourra, même en escomptant les jours de basses eaux extrĉmes et les crues extraordinaires, produire régulièrement 45 ooo chevaux utiles, et par les eaux moyennes un supplé ment de 5000 chevaux ou 3400 kilowatts.

D'autre part, les experts croient à la possibilité de cons. truire des réservorrs sur les premiers contreforts des Vosges où l'eau serait refoulée pendant la nuit pour utiliser la chute de jour, aux heures de forte consommation, sous forme d'énergie électrique. On prévoit en effet que la force livrée à l'abonnement sera essentiellement de la force de jour pour les nom breuses fabriques (filatures et tissages) de Mulhouse et des environs.

Le devis de ce projet, qui est patronné par la Sociétét industrielle de Mulhouse, a été fixé par les experts à 43 millions de marks (60 500000 francs), dont 23 pour frais de construction proprement dits, et 20 pour frais divers (acquisition de terrain, achat de la Centrale à vapeur de Mulhouse, extension du réseau des câbles, installation des transformateurs, etc.). La concession n'a pas encore été accordée.

\section{Les meilleures rivières du bassin de la Seine}

Après avoir fait connaître mon premier Lexique des rivières, celui de la Loire (2), paru en 1908, La Houille Blanche veut bien me permetlre de présenter moi-même le second, celui du bassin de la Seine.

Nous n'ignorons pas qu'il existe des projets de transport d'énergie de la vallée du Rhône à Paris même, celui de MM. Blondel, Harlé et Mahl, pour n'en citer qu'un, dont la maquette figurait à l'Exposition d'Electricité de Marseille, dans la galerie des applications de l'Electricité (3). Je sulis de ceux qui croient à la réalisation plus ou moins éloignée de ce vaste projet, mais il ne faut pas oublier que des influences locales soulèveront sans doute des oppositions à ce prélèvement de force régionale en faveur de la capltale; d'un autre côté, il faudra un effort de capitaux, dont une Métropole seule est capable. L'avenir prononcera.

Toutefois, mème en admettant que quelques centres importants, échelonnés sur ce long trajet d'envion 450 kilo. mètres, obtiennent au passage quelque peu des 80.000 chevaux prévus, le reste sera plus que sûrement absorbé en entier par Paris. D'autres petits centres, de modestes chefs lieux de canton, des communes, des bourgades même, onl cependant les mêmes droits aux bienfaits de l'hydro-életricité, et les ressources de certains cours d'eau à faible pente, peuvent souvent leur suffire. Ainsi que nous allons le voir, beaucoup l'ont déjà compris et appliqué.

(I) Les dimensions de l'écluse sont plus grandes que celles prévucs à Lat fenbourg et à Angst-Wyhlen parce que, entre Bâle et Strasbourg, on se set de remorqueurs à roues d'un déplacement plus considérable que les remor queurs à chaîne qui seront employés en amont de Bâle.

(2) La Hou lle Blanche, jullet 1908.

(3) Voir La Houille Blanche, de mai 1909. 
Tel est le but des Lexiques que je dresse, avec l'espoir d'arriver un jour à un ouvlage plus complet: un Dictionnaire des rivières de France. Si les lecteurs de La Houille Blanche ont pu voir, à l'occasion du Lexique de la Loire, qu'elles étaient les bases adoptées pour ce lexique, je leur apprendrai ici celles que je prévois pour le Dictionnaire: to pour relever plus de rivières, j'abaisse à 10 chevaux le critérium du moteur hydraulique ayant existé sur une rivière (au lieu des 25 chevaux du lexique); $2^{\circ}$ j'y ajoute encore les cours d'eau dont le débit serait de 300 litres à létlage, estimation des états consultés. mais sous toute réserve, bien entendu ; $3^{\circ}$ je joins quelques autres indicalions de perméabilité, de pluviométrie, surface du bassin; 4' enfin, les localités riveraines sont citées à partir de 300 habitants (au lieu de 1000 , dans le lexique). peu importantes, puisque j'en aurai eu la preuve dans mon ouvrage détaillé sur ces mêmes emplois dans la région normande (1), on peut émettre les différentes explications suivantes: estimation trop timide des débits des cours d'eau par l'Administration (dans le bassin de la Seine, sur les 38 cas précités au-dessous de 25 chevaux, 4 seulement auraient recours à des moteurs auxiliaires). L'emploi des turbines permet l'utilisation complète de chutes impraticables avec les anciens moleurs hydrauliques; tel est déjà le cas sur plusieurs petits cours d'eau de la Haute-Loire. Les accumulateurs électriques peuvent souvent remplacer les moteurs de secours et venir en aide à la chute; il est regrettable de ne pas mieux connaitre la proportion des deux énergies, hydraulique et vapeur, employées simultanément (2).

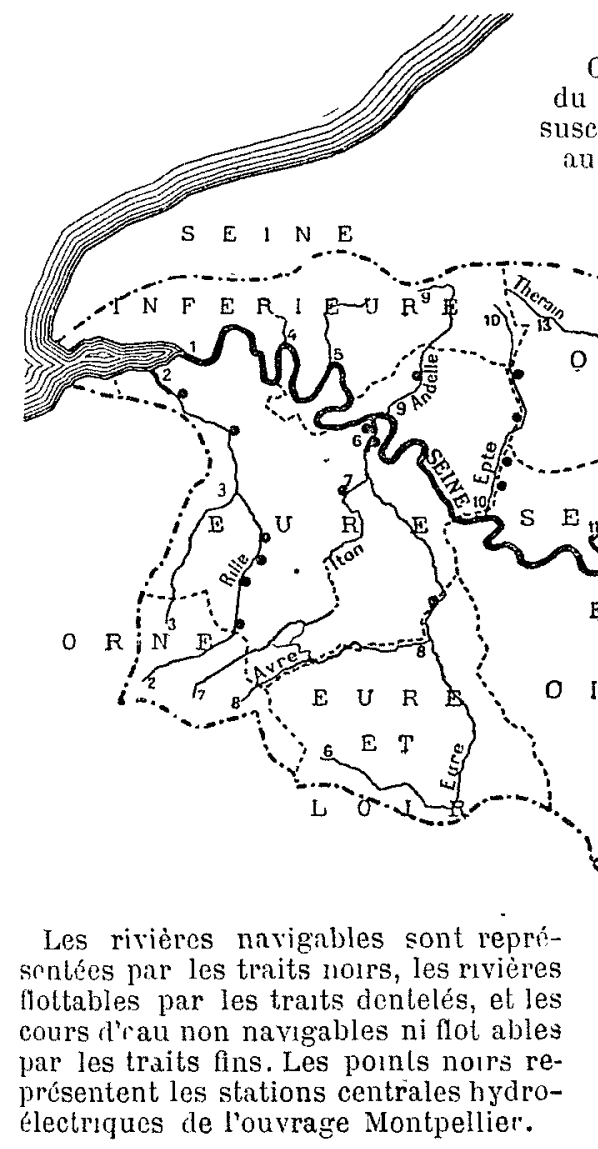

On pourra mobjecter que des rivières d'aussi faible importance ne peuvent guère présenter d'intérêt au point de vue hydro-électrique; je répondrai, comme dans la Revue Electrique du 15 janvier 1909 , par les chiffres suivants, conséquence de l'ouvrage de M. Montpellier, sur les Distributions publiques d'énergie en France, en 1907.

Sur 113 distributions de cette sorte, utilisant des cours d'eau du bassin de la Seine, 38 n'ont pu être comprises dans le présent Lexique, les rivières dont elles employaient les chules $n$ y figurant pas. C'est là, je pense, un argument ipréfutable en faveur des cours deau de moins de 25 che. vaux que relèverait le Dictionnaire projeté. De plus en plus fort, parmi ceux-ci (moins de 25 chevaux), 14 ne sont même pas compris dans la sélection des rivières.opérée à partir de 10 chevaux !

Pareillement pour le bassin de la Loire, j'avais pu comprendre 58 distributions, mais j’avais été contraint d'en laisser 31 , utilisant des cours d'eau estimés entre 10 et 25 chevaux, et même 19 ne trouvaient pas leur place parmi ceux-ci (donc rivières estimées moins de 10 chevaux.

Dans cet article, je ne puis que rechercher très brièvement les motifs probables d'un fait aussi étonnant. Faisant la part d'erreurs possibles dans toute statislique, erreurs

Sil est opportun de faire quelques réserves, quant à la source à laquelle nous avons dù puiser nos renseignements sur les usines hydro-électriques des hassins dójà étudiés, il faut reconnaitre qu'une statistique officielle, répondant aux diverses hypothèses émises ci-dessus, donnerait,à n'en pas douter, satisfaction à tous ceux qui s'intéressent au développement de la science électrique, et ils sont légion à l'heure actuelle.

Sur la table ci-jointe, le chiffre qui sc trouve à gauche de chaque rivière indique le numćro par lequel celle-ci est désignée sur la carte. Les chiffres qui se trouvent à droite indi-

(1) II. Bresson. La Houille Verte. Mise on valeur des moyennes et basses chutes de France.

(2) Ainsi, Gisors est plutôt une usine à rapeur sccourue par ane turbine hydraulique qui charge les accumulateurs jour et nuit. 
quent les rivières de plus grande importance qu'il faut remonter pour arriver à ce cours d'eau. Ainsi la vesle est numérotée 16 sur la carte. On la trouvera rapidement en r'emontant successivement la Seine (1) jusqu'à l'Oise (11), celle-ci jusqu'à l'Aisne (15), et cette dernière jusqu'à la Vesle (16).

TABLE METHODIQUE

\begin{tabular}{|c|c|c|c|c|c|}
\hline i & Snine & 0 & 26 & Petit-Gland & 1.11 .25 \\
\hline 2 & Rulle & 1 & 27 & Narne & 1 \\
\hline 3 & Charentonne & 1.2 & 28 & Grand-Morin & 1.27 \\
\hline 4 & Ste-Austreberthe & 1 & 29 & Mau & 1.27 \\
\hline 5 & Cailly & 1 & 30 & Saulx & 1.27 \\
\hline 6 & Eure & 1 & 31 & Ornain & 127.30 \\
\hline 7 & Ilon & 1.6 & 32 & Ilaise & 1.27 \\
\hline 8 & Avre & 1.6 & 33 & Blasseron & 1.27 .32 \\
\hline 9 & Alıỉelle & f & $3 i$ & Rognon & 127 \\
\hline 10 & Epte & 1 & 35 & Sneurre & 1.27 .34 \\
\hline 11 & Oise & 1 & 36 & Manoise & 1.27 .3135 \\
\hline 12 & Nonelle & 1.11 & 37 & Suize & $1.2 \overline{1}$ \\
\hline 13 & The ram & 1.11 & 38 & Essonne & 1 \\
\hline 14 & Altomne & 1.11 & 39 & Inine & 1.38 \\
\hline 15 & trsne & 1.11 & 40 & Bierville & $1.38 \quad 39$ \\
\hline 16 & Vesle & 1.11 .15 & 41 & Yonne & 1 \\
\hline 17 & Sulpe & $1.11 \quad 15$ & 42 & Mondereau & 1.41 \\
\hline 18 & Vinux & 111.15 & 43 & Armançon & 1.41 \\
\hline 19 & Aire & 1.11 .15 & 41 & Armauce & 1.41 .43 \\
\hline 20 & Agron & 1.11 .15 .19 & 45 & Landion & 1.41 .4344 \\
\hline 21 & Serre & 1.11 & 46 & Brenue & 1.41 .43 \\
\hline 22 & Vllpron & 1.11 .21 & $4^{-}$ & Serein & 1.11 \\
\hline 23 & Thion & 1.11 & 48 & Aube & 1 \\
\hline 24 & Reauregard & 1.11 & 49 & Anjm & 1.48 \\
\hline 25 & Gland & 1.11 & 50 & Ource & 1 \\
\hline
\end{tabular}

\section{L'USINE HYDRO-ÉLECTRIQUE DE BEZNAU}

Cette usine marche en parallèle avec celle de Netstall, sur la Lontsch, près de Glaris, pour alimenter le vaste réseau de distribution d'énergie électricue de la Société Motor, de Baden (Argovie), qui s'étend sur les cantons d'Argovie, de Zurich, St-Gall, Scliwytz et Glaris; elle utilise une chute de l'Aar, créée par un barrage mobile, et est construite près du village de Beznau, à peup rès à mi-chemin entre Lauffohr, où l'Aar reçoit la Limmat, émissaire du lac de Zurich, et Koblenz, oú l'Aar se jette elle-même dans le Rhin. Cette usine hy draulique contientégalement un matériel á vapeur de secours.

L'Aar est un des plus importants cours d'eau de la Suisse. Elle prend sa source aux glaciers du Finsteraarhorn, le plus haut sommet du massif de l'oberland Bernois, et reçoit les eaux des versants Est, Nord et Ouest de cet important massif. Après avoir reçu la Reuss et la Limmat, elle tranchit un court défilé, et arrive dans une vallée assez large dont elle cotoie d'abord la rive gauche, puis la rive droite jusqu'à son embouchure dans le Rhin. Son débit minimum, à Beznau, est de $195 \mathrm{~m}^{3}$. Le débit est d'environ $280 \mathrm{~m}^{3}$ pendant 300 jours, $440 \mathrm{~m}^{3}$ pendant 200 jours; en temps de crues, il peut atteindre 1.000 mètres culses. ( $\left.{ }^{*}\right)$

Aménagement hydraulique. - Ainsi que nous l'avons dit, la chute est créée par un barrage mobile, celui-ci étant étalli en amont de la courbe que décrit l'Aar à Beznau.

Le barrage de prise d'eau est constilué par sept grandes vannes métalliques, équilibrées, du type stoney, de $15 \mathrm{~m}$. d'ouverture, qui s'appuient sur six piliers en maçonneric, de $3 \mathrm{~m}$. de lar'geur, fondés en pleine rivière, et sur deux cúlées de rives. Ces vannes ont $6^{\mathrm{m}} 30$ de hauteur. Filles sont constituées par un platelage on tôle qui est maintenu rigide par des poutres horizontales a treillis. Chaque vanne est

(*) D'après Schoeiserische Banseilung suspendue par deux chaines Gall, it $6^{m} 50$ du milieu, et est équilibrée par deux contrepoids.

Afin de réduire au minimum le frottement, les vannes s'appuient sur les piliers avec interposition de galets do roulemont, ainsi que le montre les figures 1 à 3 . Létan. chéité est obtenue au moyen d'un dispositif représenté

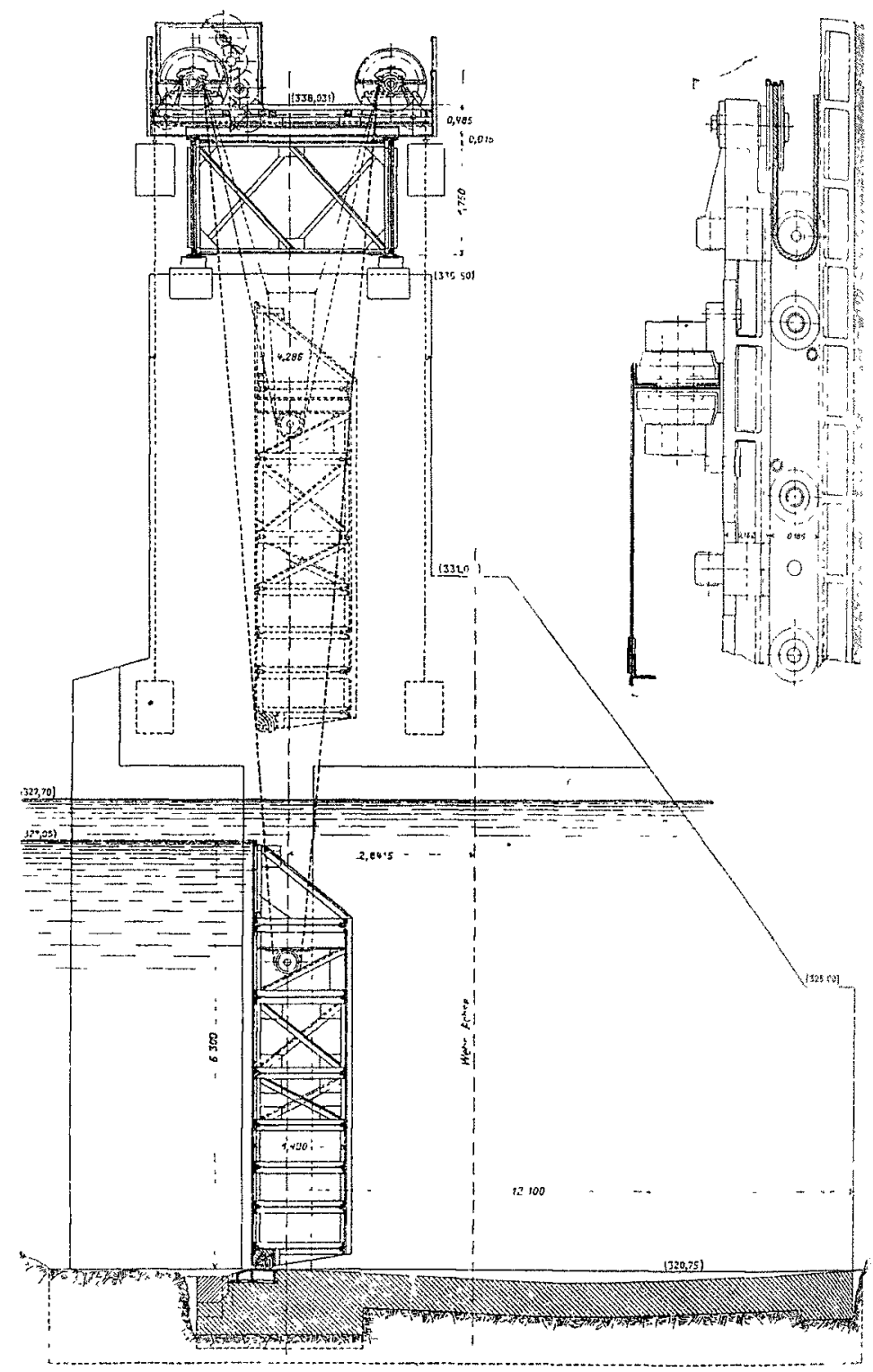

Fig. 1 el 2. - Vue en élévation d'une vanne, ot détail clu mouvement de levage suirant $C D$ de la fig. 3 .

figure 3 , tout à fait analogue à celui employé au barragge de l'usine de Séchilienne sur la Romanche (").

Le canal d'amenée coupe en ligne droite la boucle formee par l'Aar à Beznau; sa longueur est de $1180 \mathrm{~m}$., et sa

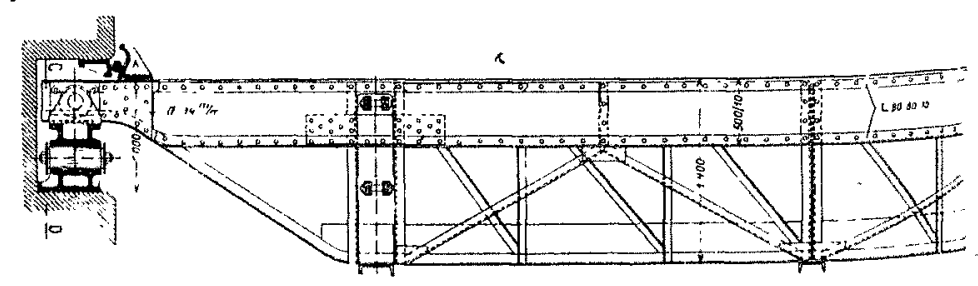

Fic. 3. - Plan d'une vanne avec son disposilif de roul ment cl destanchéite.

pente de 0,15 millimètre par mètre. Sa section transversale est celle d'un trapèze, dont la petite base a $12 \mathrm{~m}$. de largeur, et dont les côtés, perreyés, sont inclinés à deux de hase pour un de hauteur. La profondeur normale de l'eall est de 5 mètres 